\title{
Peridynamic wetness approach for moisture concentration analysis in electronic packages
}

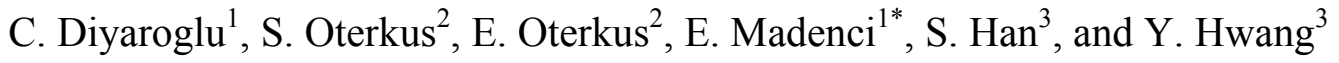 \\ ${ }^{1}$ Department of Aerospace and Mechanical Engineering, University of Arizona, Tucson, \\ AZ 85721, USA \\ ${ }^{2}$ Department of Naval Architecture, Ocean and Marine Engineering, University of \\ Strathclyde, Glasgow, UK \\ ${ }^{3}$ Samsung Electronics, Seoul, South Korea
}

\begin{abstract}
Within the finite element framework, a commonly accepted indirect approach employs the concept of normalized concentration to compute moisture concentration. It is referred to as "wetness" approach. If the saturated concentration value is not dependent on temperature or time, the wetness equation is analogous to the standard diffusion equation whose solution can be constructed by using any commercial finite element analysis software such as ANSYS. However, the time dependency of saturated concentration requires special treatment under temperature dependent environmental conditions such as reflow process. As a result, the wetness equation is not directly analogous to the standard diffusion equation. This study presents the peridynamic wetness modeling for time dependent saturated concentration for computation of moisture concentration in electronic packages. It is computationally efficient as well as easy to implement without any iterations in each time step. Numerical results concerning the one-dimensional analysis illustrate the accuracy of this approach. Moisture

\footnotetext{
${ }^{*}$ Corresponding author. Phone: 00+1+520-621-6113. Fax: 00+1+520-621-8191.

E-mail address: madenci@email.arizona.edu.
} 
concentration calculation in a three-dimensional electronic package configuration with many different material layers demonstrates its robustness.

Keywords: Concentration, moisture, peridynamics, wetness

\section{Introduction}

The components of Integrated Circuit (IC) devices are susceptible to moisture absorption at different stages of the production environment. The differential swelling between the polymeric and nonpolymeric materials, and among the polymeric materials exacerbates the hygrothermal stresses during the surface mounting (solder reflow) process in which the temperature of the IC package increases from room temperature to about $220{ }^{\circ} \mathrm{C}$. Since the temperature at this stage is higher than the glass transition temperature of polymeric materials, their mechanical properties degrade significantly. Thus, coupled with the vapor pressure in micro voids, hygro-mechanical and thermomechanical stresses may cause delamination, and subsequent cracking at different interfacial sites within the electronic package. Delamination or cracking is one of the primary failure mechanisms in plastic IC packages and often lowers the threshold for other mechanical, and electrical failures. The hygro-mechanical stresses induced through moisture pre-conditioning or pre-baking are significant compared to the thermomechanical stresses induced during the solder reflow. Combination of these stresses can be detrimental to the reliability of the IC packages.

JEDEC standard (J-STD-020) [1] defines the necessary baking procedures to remove the trapped moisture in order to prevent cracking and delamination failures during the reflow process. The bake-out times were established based on weight gain/loss measurements and one-dimensional diffusion analysis for a homogeneous medium. Such measurements require undesirable long test times (192 or 168 hours); however, they can also be used to establish accelerated equivalent conditions for moisture preconditioning. 
The moisture absorption/desorption induced failures in IC packages is more crucial for the durability of three-dimensional (3D) electronic packages with through-silicon via (TSV), ultrathin and multi-die stacking technology. However, techniques for in-situ measurement of moisture concentration within electronic packages do not exist due to the small length scales. Furthermore, the comparison of the weight gain of IC packages may not correlate with a moisture concentration inside the IC packages [2-4]. Also, Kitano et al. [2] demonstrated that the package cracking is not controlled by the absolute weight gain of packages, but the local moisture concentration at the critical interface. Therefore, it is of critical concern to compute the extent of moisture content in electronic packages.

Consequently, numerical simulation techniques have become an essential part of electronic packaging design and manufacturing for acceptable reliability. Many commercially available finite element programs are capable of solving the diffusion equation. The field variable must be continuous within the domain as a result of the nodal continuity requirement of Finite Element Method (FEM). However, it is worth noting that the saturated concentration of each material is different. Therefore, the direct solution of moisture concentration with FEM using traditional elements is not possible because continuity of concentration is required at the interface nodes. This requirement is satisfied by considering a normalized concentration (wetness) parameter introduced by Wong et al. [5]. The "wetness" parameter is the ratio of moisture concentration to its saturated moisture concentration. It is continuous across material interfaces. In FEM, the wetness analysis is performed through the concept of thermal-wetness analogy. By adjusting the thermal diffusion parameters properly, the thermal diffusion capability of a commercial finite element program can be utilized to solve for wetness if the saturated concentration value is not dependent on temperature or time. However, certain components of electronic packages are highly dependent on temperature under reflow process [6]. Most polymer materials in electronic packages have lower glass transition temperature than the reflow temperature. Hence, their $C_{\text {sat }}$ values become time dependent during the reflow process. 
The time dependency of saturated concentration requires special treatment because the wetness equation is not directly analogous to the standard diffusion equation. A finite element model of multi-material system using wetness approach under transient loading was first proposed by Wong et al. [7]; it is referred to as "piecewise normalization" approach. However, it requires several load steps and complex algorithms. In order to achieve computational efficiency and easy implementation, Wong [8] introduced an alternative approach referred to as "internal source" approach. This approach invokes temperature dependency of saturated concentration as an internal source function, and its time dependency is approximated with the backward Euler method. However, its solution with FEM is highly dependent on number of iterations performed during each time step. Recently, Wong and Park [9] revised the internal source technique, and removed the requirement for iterations.

An alternative to the FEM is peridynamics. It is originally introduced as the reformulation of continuum mechanics equations by Silling [10] to predict cracking and damage evolution. Peridynamics (PD) is extremely suitable to model discontinuities such as cracks and interfaces because its governing equation does not include any spatial derivatives; thus remaining valid regardless of discontinuities. Moreover, it is not limited to the solution of mechanical field equations and can be used to describe other governing field equations as presented by Madenci and Oterkus [11].

Hence, this study presents a peridynamic wetness modeling to determine moisture concentration in electronic packages with complex structure of dissimilar materials. It accommodates interfaces naturally while considering the effect of different material properties. Also, it does not require additional continuity conditions of moisture concentration across the interface. Moreover, the solution to peridynamic equation of wetness does not require iterations during each time step even with time dependent $C_{\text {sat }}$ values. Numerical results demonstrate that peridynamics can accurately predict the moisture diffusion in electronic packages during absorption and desorption. 


\section{Classical moisture diffusion equation}

As derived by Wong [8], the transient moisture diffusion can be expressed as

$C(\mathbf{x}, t)=D(t) \nabla^{2} C(\mathbf{x}, t)+\Theta_{m}(\mathbf{x}, t)$

where $C$ is the moisture concentration, $\Theta_{m}$ is the internal source function, $D$ is the diffusivity, $\nabla^{2}$ is the Laplace operator, and 'dot' denotes time derivative. The saturated concentration, $C_{\text {sat }}$ is defined as

$C_{s a t}=S P_{V P}$

where $S$ is the solubility and $P_{V P}$ is the ambient vapor pressure. The diffusivity and solubility can be defined through the Arrhenius law as

$D(t)=D_{0} e^{\left(\frac{-E_{D}}{R T(t)}\right)}$

and

$S(t)=S_{0} e^{\left(\frac{E_{S}}{R T(t)}\right)}$

in which $D_{0}$ is the diffusivity factor, $E_{D}$ is the activation energy of the diffusivity, $S_{0}$ is the solubility factor, $E_{S}$ is the activation energy of the solubility, $R$ is the universal gas constant ( $R=8.3145 \mathrm{~J} / \mathrm{Kmol}$ ), and $T$ represents temperature.

Finite Element Method (FEM) is capable of solving for the moisture concentration, Eq. (1) if the domain is homogeneous with nodal continuity. However, it breaks down in 
the case of a nonhomogeneous domain in which moisture concentration is not continuous at the interface of different materials. In order to resolve this issue, Wong et al. [5], introduced a normalized parameter called "wetness" as

$$
w=\frac{C}{C_{\text {sat }}}
$$

The interface continuity can be assumed in the form

$$
w_{1}=\frac{C_{1}}{C_{\text {sat } 1}}=w_{2}=\frac{C_{2}}{C_{\text {sat } 2}}
$$

where the subscripts indicate different materials on both sides of the interface. Therefore, the interface continuity of these normalized variables is automatically satisfied. The value of unity indicates full saturation, and a zero value indicates no moisture concentration. The wetness approach ensures the equalization of chemical potentials at the interface of dissimilar materials which is not satisfied by using a direct concentration approach [8].

With the "wetness" parameter, Eq. (1) can be recast for time independent $C_{\text {sat }}$ as

$w(\mathbf{x}, t)=D(t) \nabla^{2} w(\mathbf{x}, t)$

This equation can be solved readily by employing the thermal-wetness analogy as proposed by Wong et al. [5]. Its solution can be readily achieved by existing techniques such as any finite element method.

If $C_{\text {sat }}$ is time (temperature) dependent, Eq. (1) can be recast in terms of the "wetness" parameter as [8] 
$C_{\text {sat }}(t) w(\mathbf{x}, t)=D(t) C_{\text {sat }}(t) \nabla^{2} w(\mathbf{x}, t)+\Theta_{m}(\mathbf{x}, t)$

with

$\Theta_{m}(\mathbf{x}, t)=-w(\mathbf{x}, t) C_{s a t}(t)$

However, the solution to this equation requires a special method such as the "piecewise normalization" approach by Wong et al. [7] or the "internal source" approach by Wong [8]. The latter approach is straightforward and easy to implement. However, it is highly dependent on the number of iterations performed during each time step because $\Theta_{m}(\mathbf{x}, t)$ is assumed as constant between the consecutive time steps. The diffusivity parameter and the saturated concentration are also updated in each time step as part of this procedure.

\section{Peridynamic form of wetness equation}

The local form of the moisture diffusion equation, Eq. (1) can be rewritten in its nonlocal peridynamic (PD) form as [12]

$\frac{\partial}{\partial t}\left[C_{s a t}(t) w(t)\right]=\int_{H} f\left(w^{\prime}, w, \mathbf{x}^{\prime}, \mathbf{x}, t\right) d V^{\prime}+\Theta_{m}(t)$

where $f\left(w^{\prime}, w, \mathbf{x}^{\prime}, \mathbf{x}, t\right)$ is the moisture concentration response function. It enables the exchange of moisture between material points $\mathbf{x}$ and $\mathbf{x}^{\prime}$ that are connected through hygro-bonds. In peridynamics, the interaction between material points is nonlocal, and a material point is influenced by the other material points within its neighborhood defined by the interaction region, $H$ as shown in Fig. 1. The size of this interaction region is defined by its radius, $\delta$, referred to as the PD "horizon". 


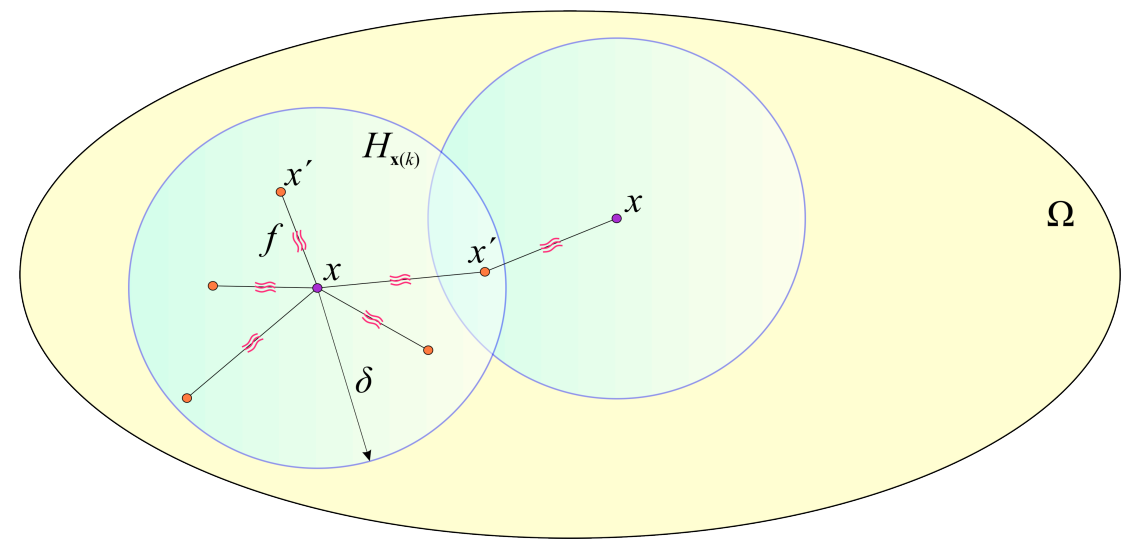

Fig. 1. Interaction of material point $\mathbf{x}$ with its neighboring point, $\mathbf{x}^{\prime}$

The moisture concentration response function can be defined as

$f\left(w^{\prime}, w, \mathbf{x}^{\prime}, \mathbf{x}, t\right)=d(t) \frac{w\left(\mathbf{x}^{\prime}, t\right)-w(\mathbf{x}, t)}{\left|\mathbf{x}^{\prime}-\mathbf{x}\right|}$

The PD bond parameter, $d(t)$ in Eq. (9) can be expressed in terms of the classical diffusivity, $D(t)$ and the saturated concentration, $C_{s a t}(t)$. By equating the peridynamic and classical form of diffusion equations for a linear concentration variation as the PD horizon, $\delta$ (radius of the PD interaction region, $H$ ) approaches zero leads to [12]

$d=\frac{2 D C_{s a t}}{A \delta^{2}}(1-\mathrm{D}), d=\frac{6 D C_{s a t}}{\pi h \delta^{3}}(2-\mathrm{D})$ and $d=\frac{6 D C_{s a t}}{\pi \delta^{4}}(3-\mathrm{D})$

where $A$ is the cross-sectional area of a one-dimensional structure and $h$ is the thickness of a two-dimensional structure. Note that, the response function, $f$ is zero for material points outside the horizon; i.e., $\left|\mathbf{x}^{\prime}-\mathbf{x}\right|>\delta$.

In discrete form, the integral term involving the response function is replaced by a finite summation; thus, the diffusion equation can be rewritten as 
$C_{s a t} \frac{\partial w_{(k)}}{\partial t}=\sum_{j=1}^{N} f_{(k)(j)}\left(w_{(j)}, w_{(k)}, \mathbf{x}_{(j)}, \mathbf{x}_{(k)}, t\right) V_{(j)}+\Theta_{m(k)}$

with

$\Theta_{m(k)}=-w_{(k)} \frac{\partial C_{s a t}}{\partial t}$

where the displacement $w_{(k)}$ and $w_{(j)}$ are wetness values at material points $\mathbf{x}_{(k)}$ and $\mathbf{x}_{(j)}$, respectively. The response function $f_{(k)(j)}$ represents the wetness exchange between material points $\mathbf{x}_{(k)}$ and $\mathbf{x}_{(j)}$. The summation range $N$ is the number of family members of material point $\mathbf{x}_{(k)}$. The family of material point $\mathbf{x}_{(k)}$ is denoted by $H_{\mathbf{x}(k)}$ as shown in Fig.1.

Unlike the FEM, peridynamics permits the specification of different interface properties. As shown in Fig. 2, material points, $\mathbf{x}_{(k)}$ and $\mathbf{x}_{(j)}$, are located on opposite sides of the interface with diffusion coefficients $D_{(k)}$ and $D_{(j)}$ and saturated concentration $C_{s a t(k)}$ and $C_{s a t(j)}$ values. The peridynamic bond between material points $\mathbf{x}_{(k)}$ and $\mathbf{x}_{(j)}$ is split between material 1 and material 2. The segments of this bond in material 1 and 2 , are denoted by $l_{(k)}$ and $l_{(j)}$, respectively. The effective property of this bond between material points, $\mathbf{x}_{(k)}$ and $\mathbf{x}_{(j)}$ can be approximated as

$$
D C_{s a t}=\frac{l_{(k)}+l_{(j)}}{\frac{l_{(k)}}{D_{(k)} C_{s a t(k)}}+\frac{l_{(j)}}{D_{(j)} C_{s a t(j)}}}
$$


During the solution process, it is necessary to monitor the material properties, $D$ and $C_{\text {sat }}$, and update them accordingly under temperature dependent environmental conditions.

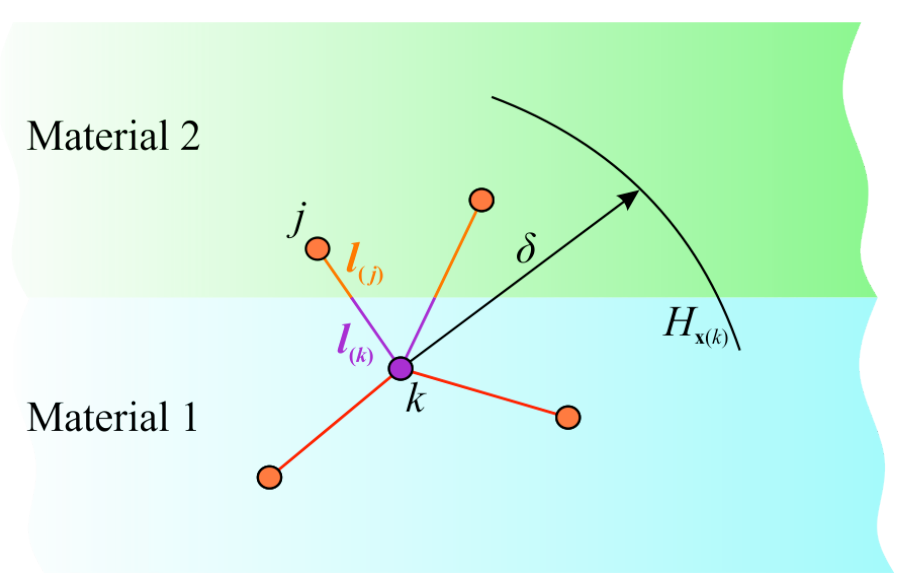

Fig. 2. PD interactions of material points near the interface region

In order to implement the saturated concentration values as boundary conditions, the fictitious boundary layer regions are created with a depth of $\delta$ in addition to the actual dimensions of a domain as described by Madenci and Oterkus [11]. During the solution process for each time step, the time dependency of $C_{\text {sat }}$ can be approximated with backward Euler method between the consecutive time steps as

$\Theta_{m(k)}=-w_{(k)} \frac{\Delta C_{s a t}}{\Delta t}=-w_{(k)} \frac{C_{s a t}(t+\Delta t)-C_{s a t}(t)}{\Delta t}$

Also, the PD bond parameter, $d(t)$ is updated in accordance with Eqs. (2) and (3a). Therefore, the solution to peridynamic wetness equation does not require any iteration during the solution procedure in order to conserve moisture concentration.

\section{Numerical results}

Numerical results first illustrate the accuracy of the peridynamic wetness modeling by considering a one-dimensional bar made of two different materials experiencing either 
absorption or desorption. Subsequently, a three-dimensional cross-stack package configuration is considered to demonstrate the current capability for moisture concentration and weight gain predictions.

\section{A. One-dimensional analysis for absorption}

A bar with a length of $L=1 \mathrm{~mm}$ is composed of two different materials as shown in Fig.

3. The lateral surfaces of the bar are isolated and the boundary conditions are specified as $C(x=-L)=C_{\text {sat } 1}$ and $C(x=L)=C_{\text {sat } 2}$. The bar is initially at a dry state, $C_{1}(x, t=0)=C_{2}(x, t=0)=0$ before the start of absorption. The time dependent temperature is uniformly distributed along the bar as

$T_{a}(t)=(25+t / 60) \mathrm{C}$

As time progresses, the temperature changes uniformly but the partial vapor pressure is kept constant as $P_{V P}=3207 \mathrm{~Pa}\left(P_{s a t}=P_{V P}\right.$ at $\left.25^{\circ} \mathrm{C}\right)$ during the absorption process. This problem was considered previously by Jang et al. [13] and the properties of each material are given in Table 1. Note that the materials have the same activation energy.

Table 1. Material properties

\begin{tabular}{lcc}
\hline & Material 1 & Material 2 \\
\hline Diffusivity factor, $D_{0}\left(\mathrm{~m}^{2} / \mathrm{s}\right)$ & $5 \times 10^{-3}$ & $4 \times 10^{-3}$ \\
\hline Solubility factor, $S_{0}\left(\mathrm{~kg} / \mathrm{m}^{3}\right) \mathrm{Pa}$ & $6 \times 10^{-10}$ & $2 \times 10^{-10}$ \\
\hline Pressure factor, $P_{0}(\mathrm{~Pa})$ & $5.0492 \times 10^{10}$ & $5.0492 \times 10^{10}$ \\
\hline Diffusion activation energy, $E_{D}(\mathrm{~J} / \mathrm{mol})$ & $5 \times 10^{4}$ & $5 \times 10^{4}$ \\
\hline Solubility activation energy, $E_{S}(\mathrm{~J} / \mathrm{mol})$ & $4 \times 10^{4}$ & $4 \times 10^{4}$ \\
\hline
\end{tabular}




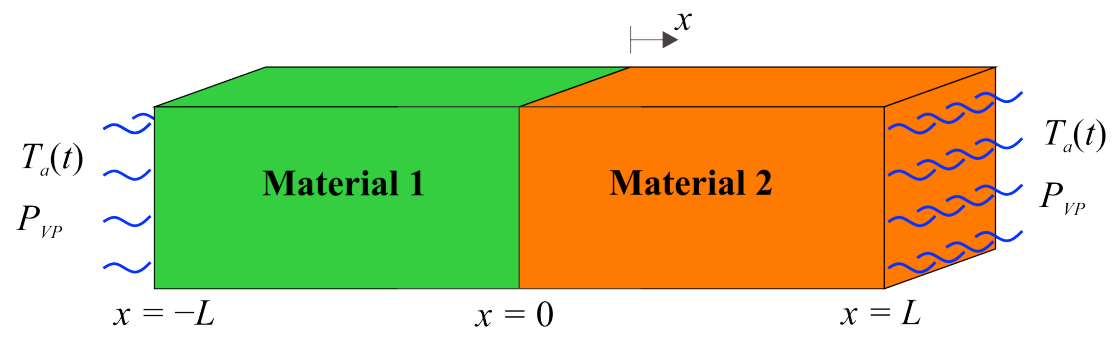

Fig. 3. A bar with a dissimilar material interface

The initial and boundary conditions in terms of the wetness parameters are specified as

$w(-L<x<L, t=0)=0$

and

$w(x=-L, t)=1$ and $w(x=L, t)=1$

As shown in Fig. 4, the PD model of the bar is discretized with spacing $\Delta x=L / 100$ between the material points, and the horizon sizes are chosen as $\delta=3.015 \Delta x$ and $\delta=1.05 \Delta x$, respectively. Its uniform cross-sectional area is specified as $A=(\Delta x)^{2}$. The PD equations are solved implicitly with a time step size of $\Delta t=2 \mathrm{~s}$.

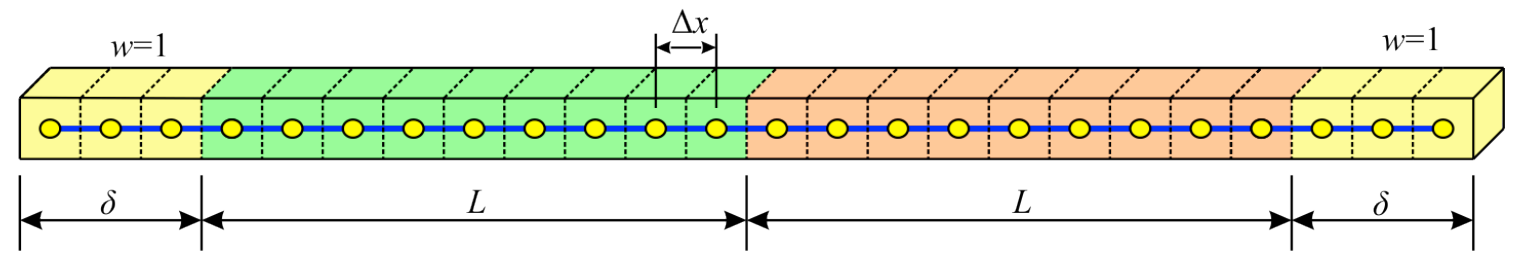

Fig. 4. Peridynamic computational domain

The moisture concentration predictions based on the "internal source" approach and peridynamics are shown in Fig. 5 at time, $t=1800$ and $3600 \mathrm{~s}$. As evident in this figure, both methods are in agreement. However, the PD predictions do not require the 
additional iteration steps between the time steps. As time progresses, the temperature increases while the saturated concentration values of both materials decrease. The moisture diffusion continues in the bar until both materials reach their saturated states. They are low compared to the room temperature levels, after $3600 \mathrm{sec}$ of absorption. Fig. 6 shows PD wetness results at time $t=1800$ and $3600 \mathrm{~s}$ for the horizon sizes of $\delta=3.015 \Delta x$ and $1.05 \Delta x$. It is apparent that the effect of horizon size is insignificant.

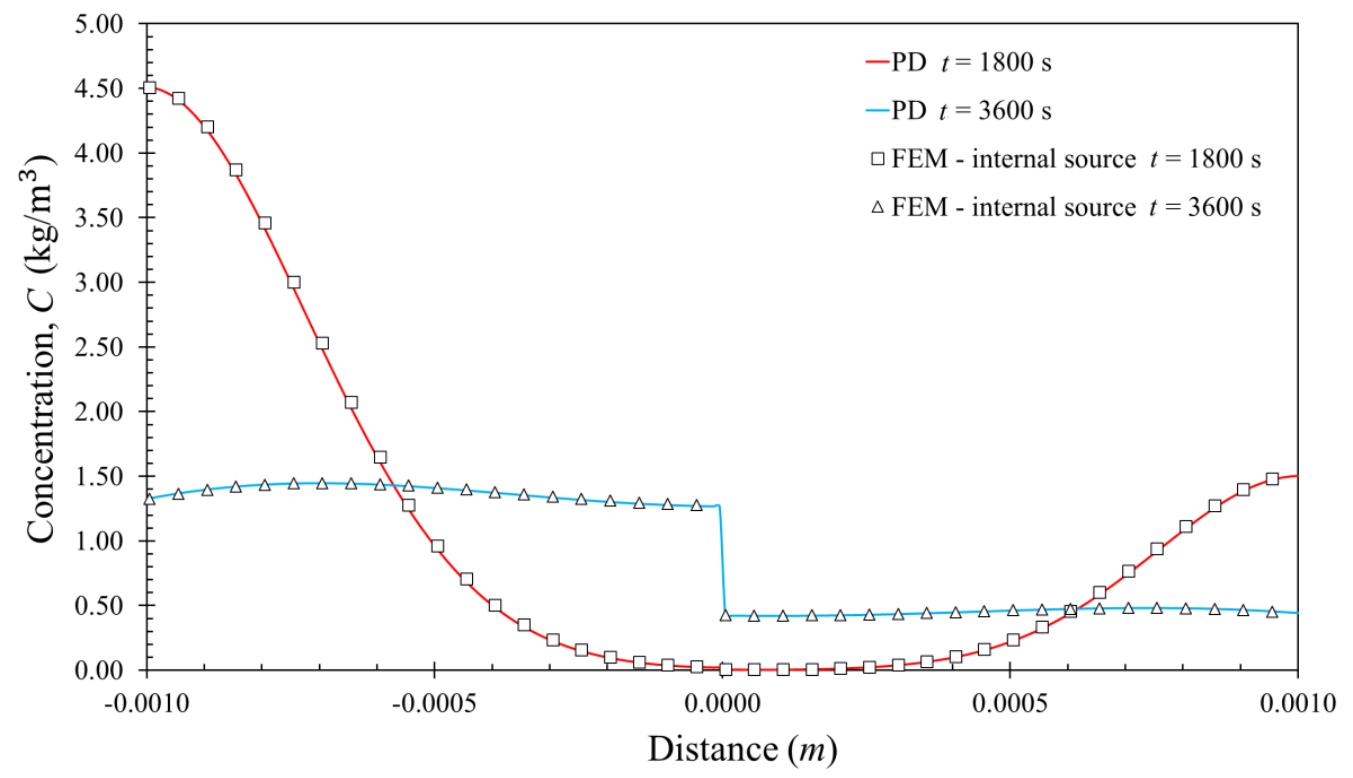

Fig. 5. Moisture concentration along the bar as time progresses

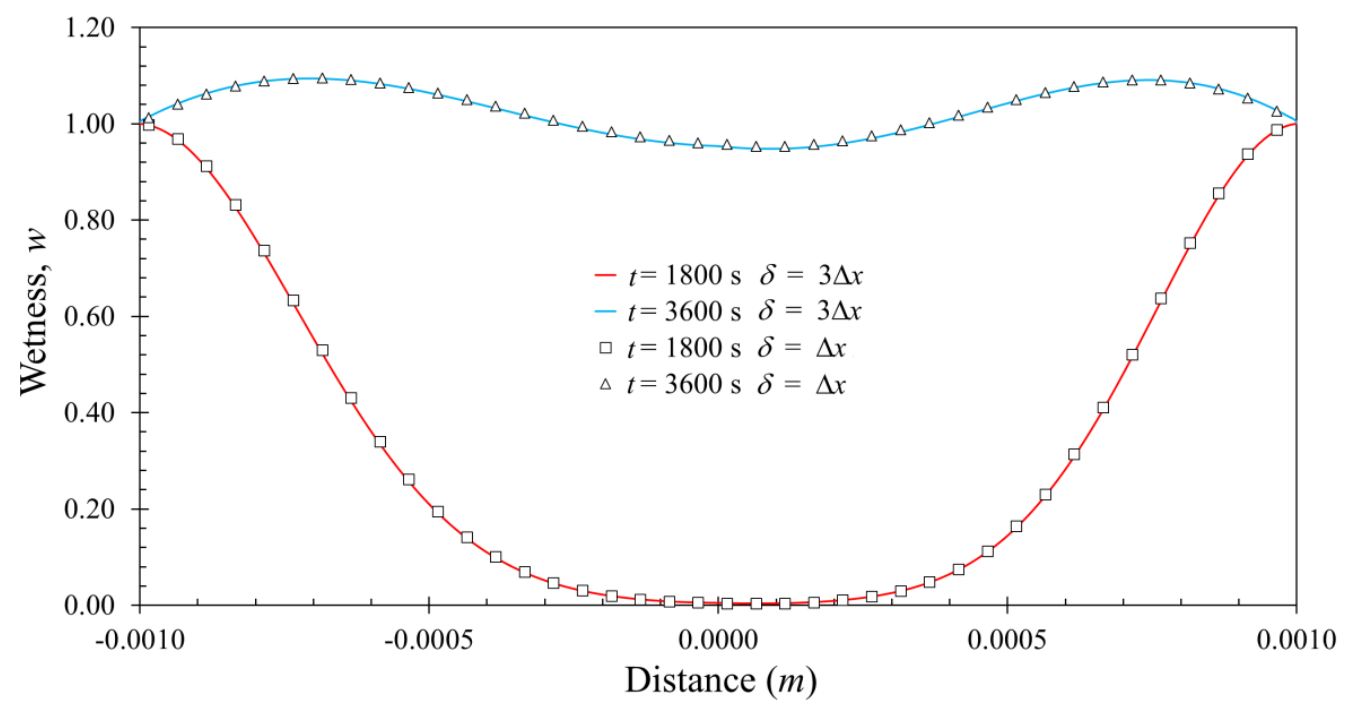

Fig. 6. PD wetness variation along the bar as time progresses for different horizon values 


\section{B. One-dimensional analysis for desorption}

The geometry, and material properties of the bar are the same as that of the previous problem. The lateral surfaces of the bar are isolated and the boundary conditions are specified as $C(x=-L)=0$ and $C(x=L)=0$. The bar is initially fully saturated at $85^{\circ} \mathrm{C} / 100 \% R H$. It is subjected to desorption process and the temperature changes uniformly with time as

$T(t)=85 \times e^{0.02 t} \mathrm{C}$

Furthermore, the solubility activation energies are continuous at the interface of materials.

The initial and boundary conditions in terms of the wetness parameters are specified as

$w(-L<x<L, t=0)=1$

and

$w(x=-L, t)=0$ and $w(x=L, t)=0$

The comparison of moisture concentration predictions based on the FEM "internal source" and peridynamics are shown in Fig. 7 as time progresses. As apparent in Fig. 7, both methods provide comparable predictions. Fig. 8 shows PD wetness predictions at time $t=20,40,50$ and $60 \mathrm{~s}$ for the horizon sizes of $\delta=3.015 \Delta x$ and $1.05 \Delta x$. It also indicates that the effect of horizon size is insignificant. As the time progresses, desorption takes place and both wetness and corresponding moisture concentration decrease throughout the bar. Moreover, it captures the expected discontinuous concentration at the interface. 


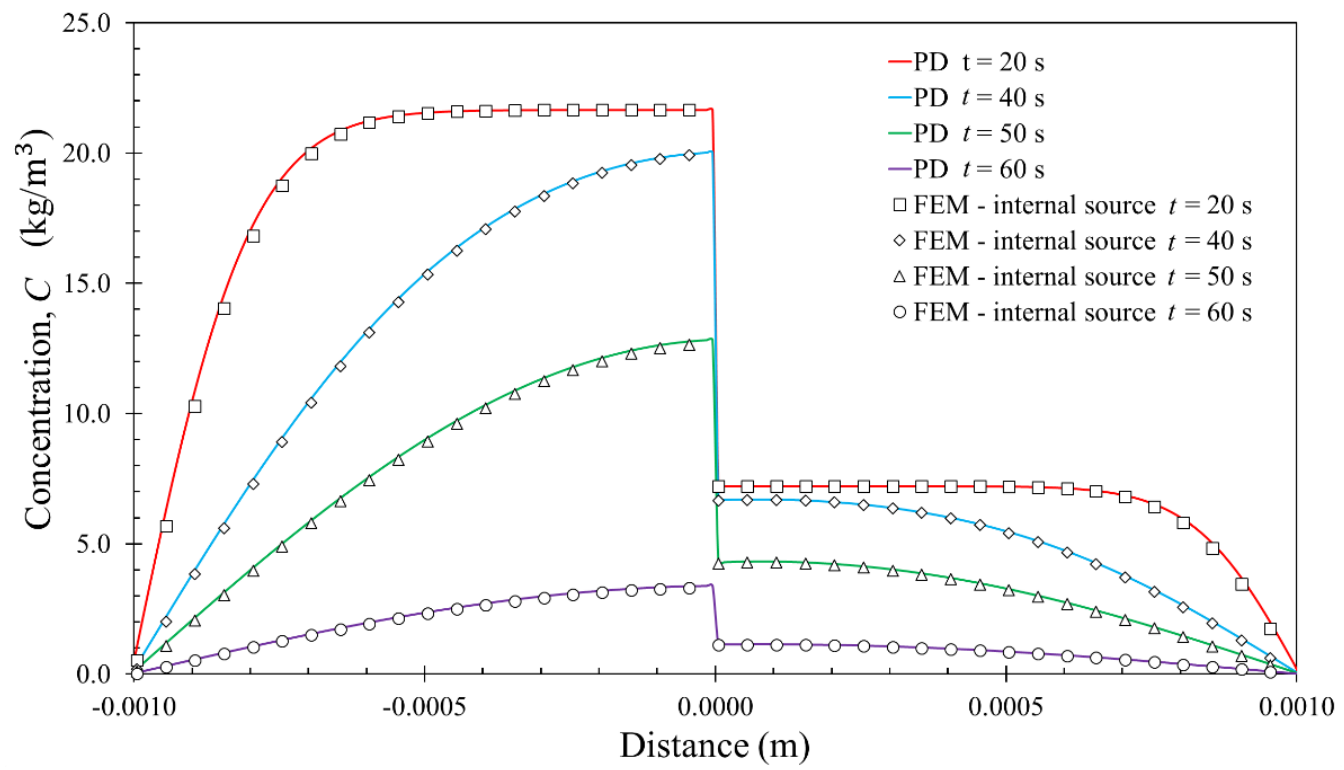

Fig. 7. Moisture concentration along the bar as time progresses

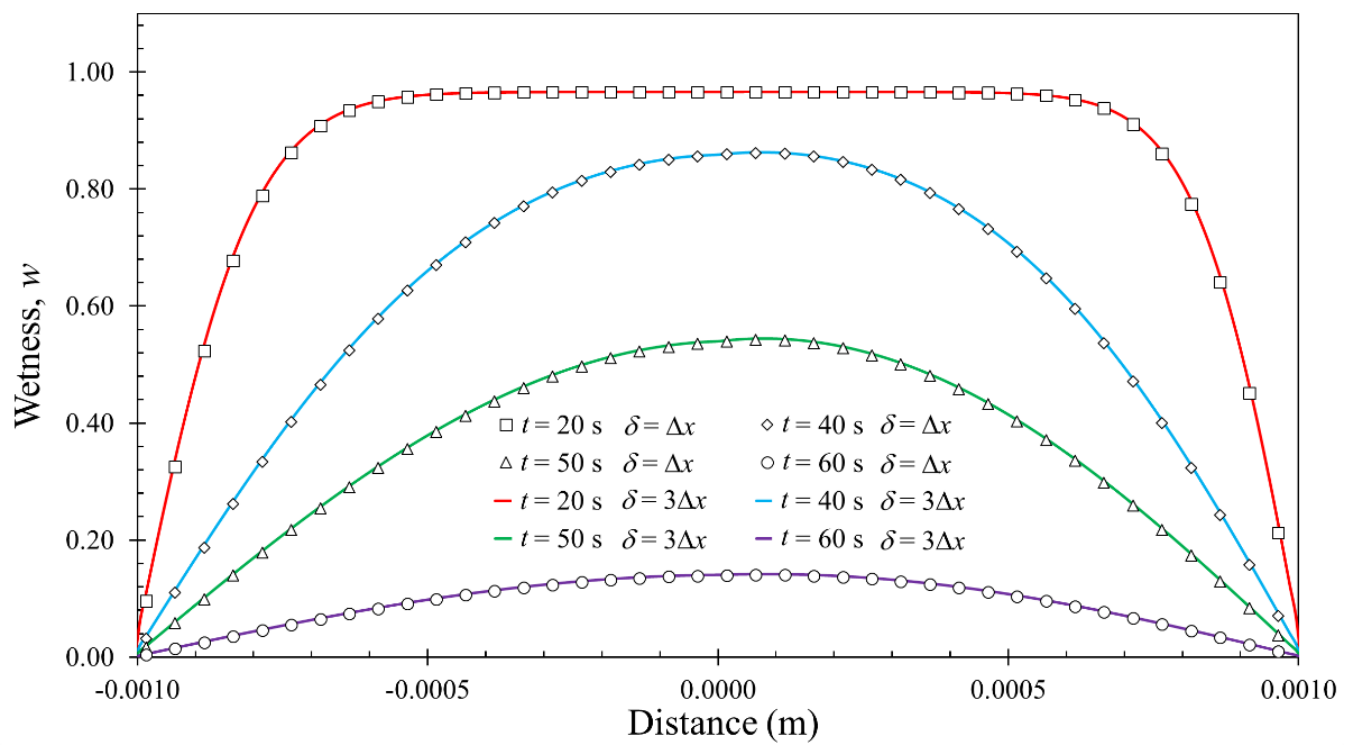

Fig. 8. PD wetness variation along the bar as time progresses for different horizon values

C. One-dimensional analysis for desorption with unequal values of $E_{S}$

The geometry, boundary conditions and material properties of the bar is the same as that of the previous problem except for the solubility activation energy of material 2. It is modified as $E_{S}=4.50 \times 10^{4} \mathrm{~J} / \mathrm{mol}$. The comparison of moisture concentration predictions based on the FEM "internal source" and peridynamics are shown in Fig. 9 at 
time $t=20,40,50$ and $60 \mathrm{~s}$. As time progresses, the moisture diffusion continues in the bar until both materials nearly lose their concentrations.

Both methods provide predictions that are in close agreement. Fig. 10 shows PD wetness predictions at time $t=20,40,50$ and $60 \mathrm{~s}$ for the horizon sizes of $\delta=3.015 \Delta x$ and $1.05 \Delta x$. The peridynamics captures the effect of time dependency of $C_{\text {sat }}$ on wetness results. The predictions are not influenced by the horizon size.

As apparent from Fig. 9 that the adjacent materials do not show proportionate change with time due to their time varying $C_{\text {sat }}$ values. However, the wetness values maintain their continuity at the interface in both approaches as shown in Fig. 10. Thus, the continuity of chemical potentials is satisfied [8]. The PD wetness equation of motion can effectively capture time dependent effect of material properties under temperature dependent environmental conditions.

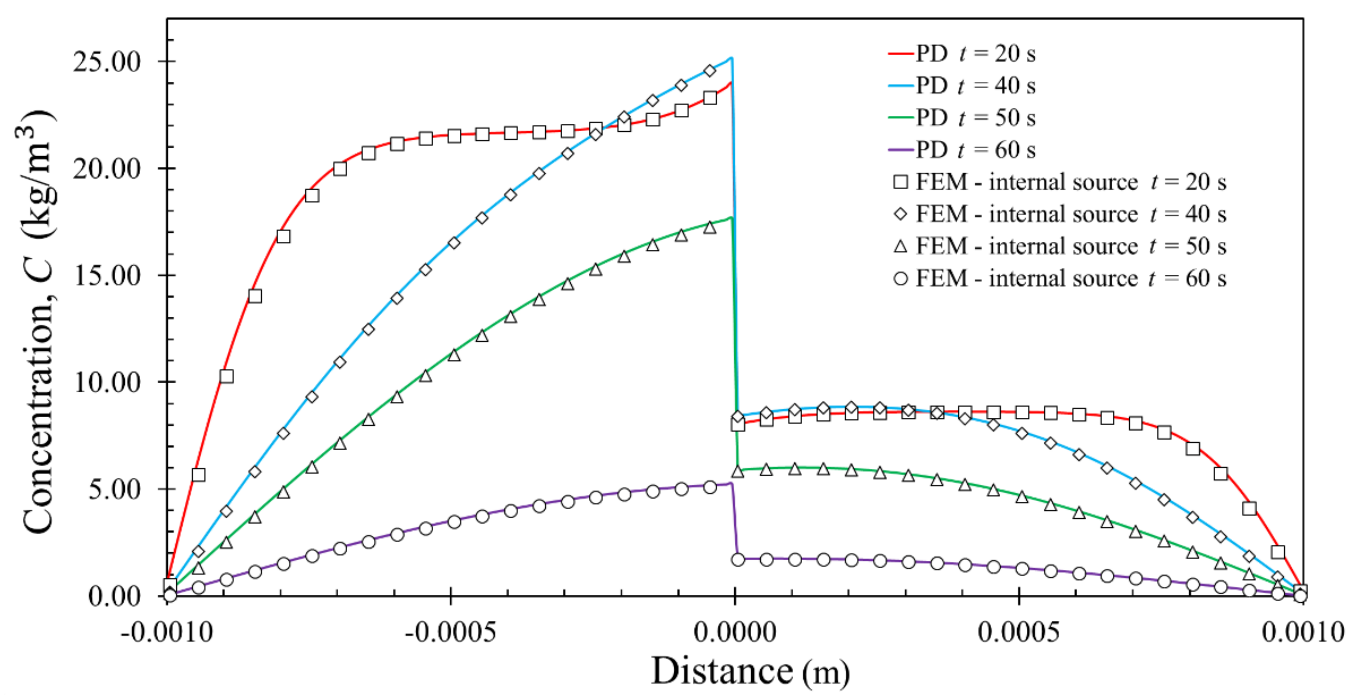

Fig. 9. Moisture concentration along the bar as time progresses 


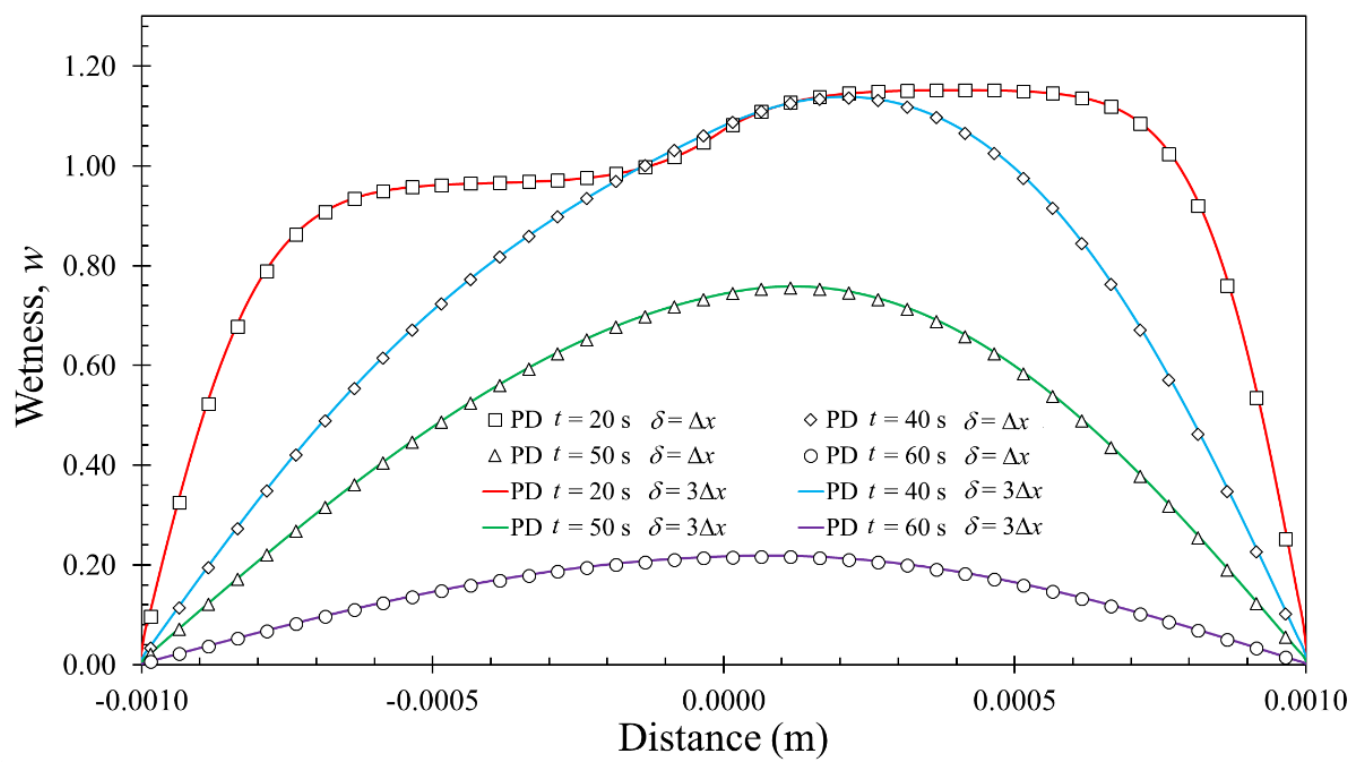

Fig. 10. PD wetness variation along the bar as time progresses for different horizon values

\section{Three-Dimensional Cross-Stack Package Configuration}

A three-dimensional cross-stack package configuration is considered as shown in Fig. 11. The parameters that describe the package dimensions are specified as $L=5 \mathrm{~mm}$ and $W=5 \mathrm{~mm}$. The length and width of the chip and DAF regions are ${ }_{c}=3 \mathrm{~mm}$ and $w_{c}=2 \mathrm{~mm}$, respectively. The thickness of the entire package, substrate, DAF and chip regions are specified as $H=2.60 \mathrm{~mm}, t_{s}=0.60 \mathrm{~mm}, t_{d}=0.10 \mathrm{~mm}$ and $t_{c}=0.60 \mathrm{~mm}$, respectively. The diffusivity constants $D_{s}, D_{e}, D_{d}$ and $D_{c}$ for the substrate, EMC, DAF, and chip, respectively, in the case of absorption phase are specified as:

$$
\begin{aligned}
& D_{s}=582.62 \times 10^{-9} \mathrm{~m}^{2} / \mathrm{hr} \\
& D_{e}=6.228 \times 10^{-9} \mathrm{~m}^{2} / \mathrm{hr} \\
& D_{d}=279.21 \times 10^{-9} \mathrm{~m}^{2} / \mathrm{hr} \\
& D_{c}=0
\end{aligned}
$$

The package is initially at dry state before the absorption process. The boundary conditions for the absorption phase are specified as 
$w(x=L / 2, y, z)=w(x, y=W / 2, z)=w(x, y, z=H / 2,-H / 2)=1$.

The saturated moisture concentration values of the materials are specified as $C_{\text {sat }, s}=4.4398 \mathrm{~kg} / \mathrm{m}^{3}, C_{\text {sat }, e}=6.1239 \mathrm{~kg} / \mathrm{m}^{3}$ and $C_{\text {sat }, d}=20.748 \mathrm{~kg} / \mathrm{m}^{3}$. The total time for the absorption phase is 400 hours with a time step size of 1 hour. The spacing between the material points is specified as equal to the thickness of the DAF region, i.e. $\Delta x=t_{d}$, and the horizon size is chosen as $\delta=1.733 \Delta x$. Only quarter of the package is modelled as in Fig. 12 due to the presence of symmetry.

The wetness and concentration distributions for the quarter section of the package at time $t=50$ hours and at the end of absorption phase are shown in Figs. 13 and 14. The weight gain during the absorption process for individual materials and the entire package is shown in Fig. 15. All materials reach their saturated state after 400 hours. The peridynamic weight gain predictions presented in Table 2 agree well with the analytical weight gain values. 
Top View
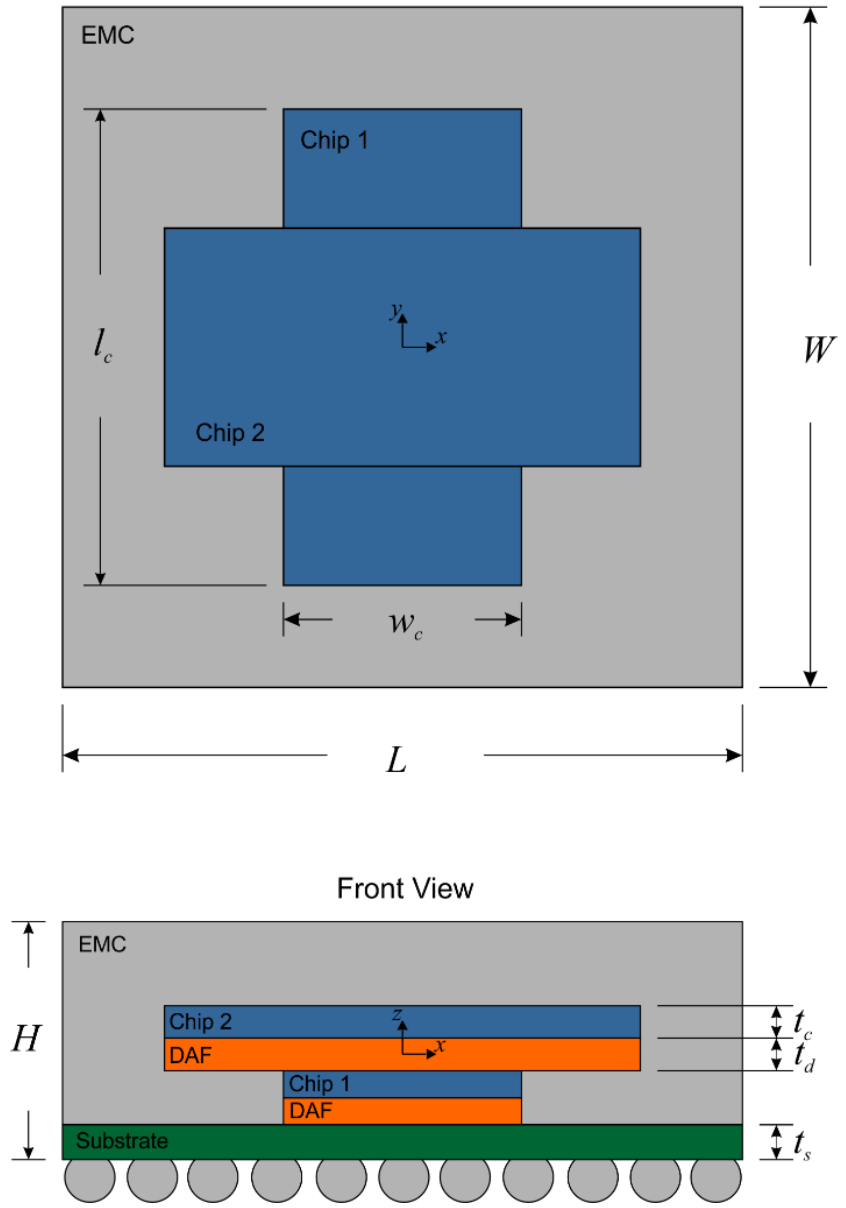

Fig. 11. Geometric parameters and architecture of the cross-stack package

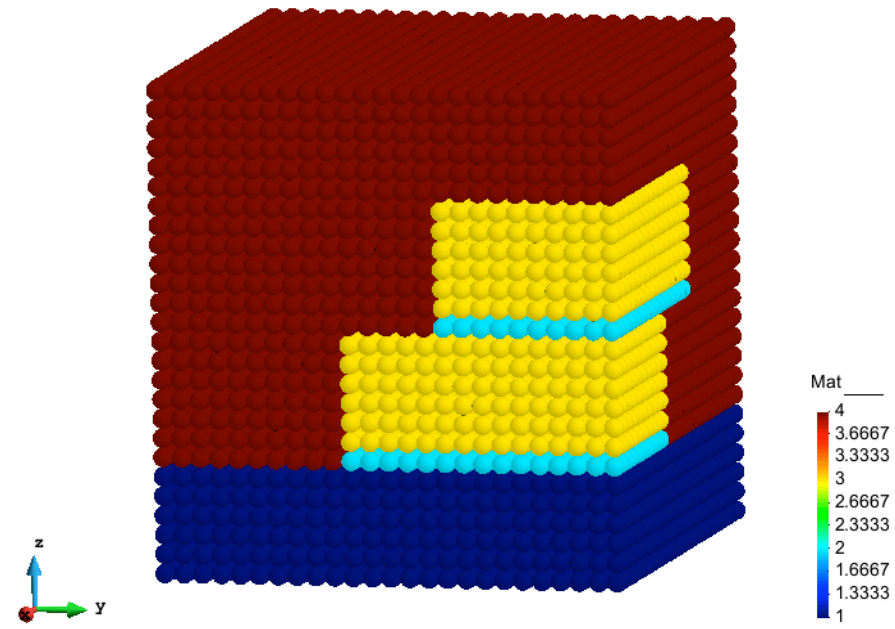

Fig. 12. PD material points. Each color represents different type of material. Material numbers: 1- Substrate, 2- DAF, 3- Chip, 4- EMC 


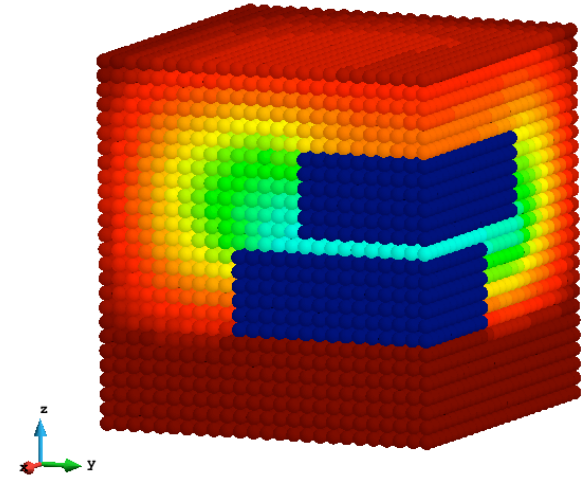

(a)

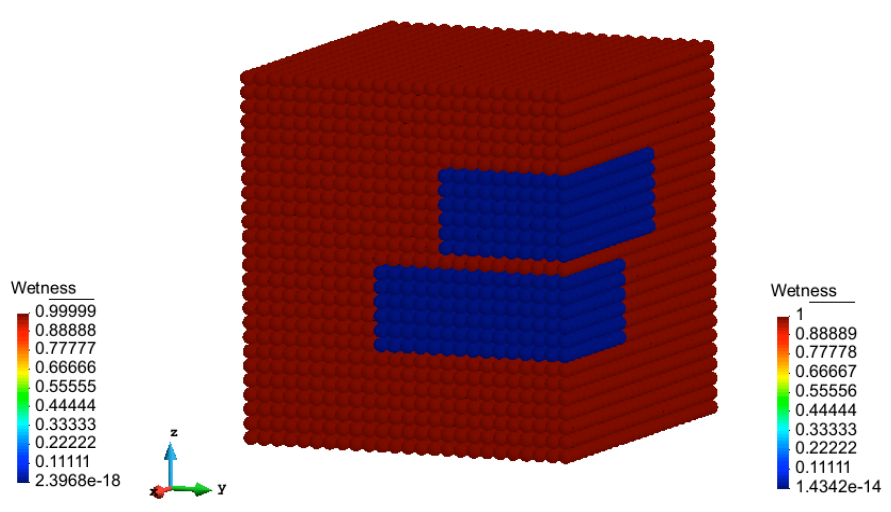

(b)

Fig. 13. Wetness distribution at (a) $t=50$ and (b) 400 hours

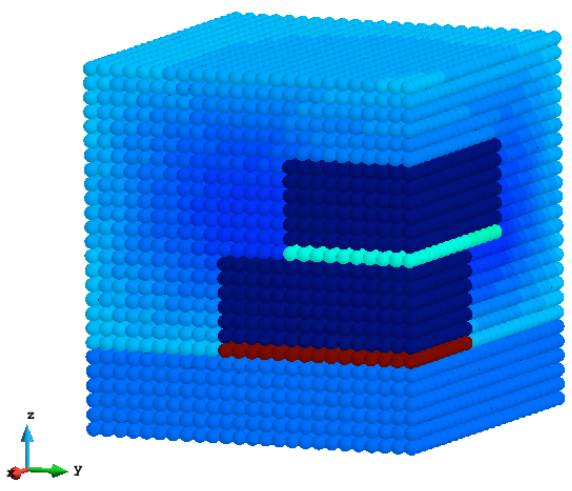

(a)
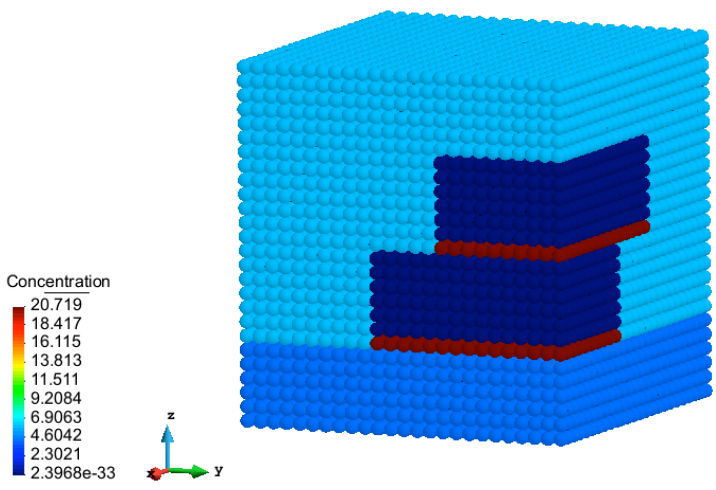

(b)

Fig. 14. Concentration values at (a) $t=50$ and (b) 400 hours

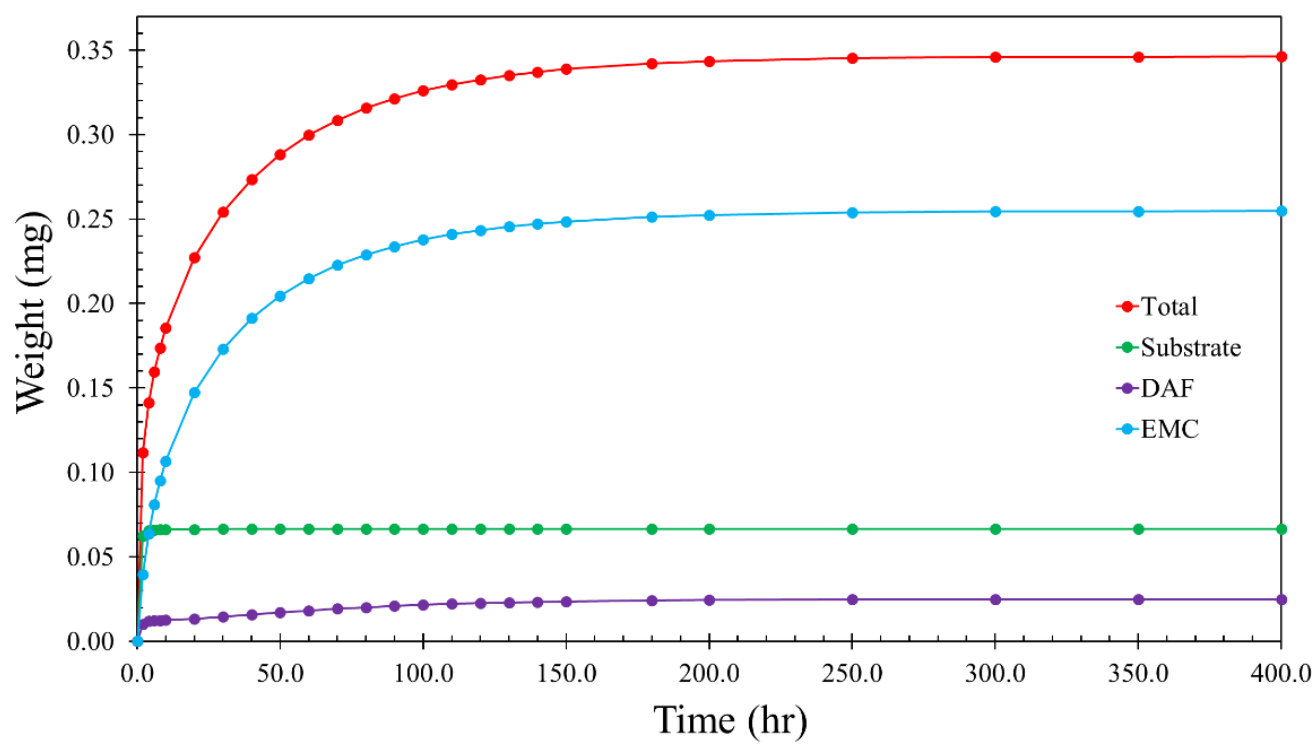

Fig. 15. Weight gain as time progresses 
Table 2. Theoretical weight gain of electronic package

\begin{tabular}{ccccc}
\hline & \multirow{2}{*}{ Volume $\left(\mathbf{m m}^{3}\right)$} & \multirow{2}{*}{$\boldsymbol{C}_{\text {sat }}\left(\mathbf{k g} / \mathbf{m}^{3}\right)$} & \multicolumn{2}{c}{ Weight $(\mathbf{m g})$} \\
\cline { 4 - 5 } & & & Theoretical & PD \\
\hline Substrate & 15 & 4.4398 & 0.066597 & 0.066597 \\
\hline DAF & $0.6 \times 2$ & 20.748 & 0.024898 & 0.024888 \\
\hline Chip & $3.6 \times 2$ & 0.0 & 0.0 & 0.0 \\
\hline EMC & 41.6 & 6.1239 & 0.254754 & 0.254705 \\
\hline Total & 65 & & 0.346249 & 0.346190 \\
\hline
\end{tabular}

\section{Conclusions}

This study presents a wetness approach to predict moisture concentration in electronic packages by using peridynamics. It enables the imposition of interface continuity conditions in a natural way because the peridynamic form of the moisture diffusion equation does not contain any spatial derivatives. Also, it provides correct results without a need of any iteration even in the presence of time dependent saturated concentrations. The capability of the current approach is demonstrated by considering simple benchmark problems, and a three-dimensional electronic package configuration with many different material layers.

\section{Acknowledgments}

This study was supported by the SAMSUNG Global Research Outreach (GRO) Program.

\section{References}

[1] J-STD-020D, 2008, "Moisture/reflow sensitivity classification for non-hermetic solid state surface mount devices".

[2] Kitano, M., Nishimura, A., Kawai, S. and Nishi, K., 1988, "Analysis of Package Cracking During Reflow Soldering Process," IEEE/IRPS, pp. 90-95.

[3] Shook, R., Vaccaro, R. and Gerlach, D., 1998, "Method for Equivalent Acceleration of JEDEC/IPC Moisture Sensitivity Levels", Annual International Reliability Physics Symposium, pp. 214-219. 
[4] Shi, D., Fan, X. and Xie, B., 2008, "A New Method for Equivalent Acceleration of JEDEC Moisture Sensitivity Levels", Proceedings of the 58th Electronic Components and Technology Conference, pp. 907-912.

[5] Wong, E. H., Teo, Y. C. and Lim, T. B., 1998, "Moisture diffusion and vapor pressure modeling of IC packaging," Proceedings of the 48th Electronic Components and Technology Conference, pp. 1372-1378.

[6] Xie, B., Fan, X. J., Shi, X. Q. and Ding, H., 2009, "Direct Concentration Approach of Moisture Diffusion and Whole-Field Vapor Pressure Modeling for Reflow Process: Part I - Theory and Numerical Implementation. ASME Journal of Electronic Packaging, Vol. 131, pp. 31010-1-7.

[7] Wong, E. H., Koh, S. W., Lee, K. H. and Rajoo, R., 2002, "Advanced moisture diffusion modeling and characterisation for electronic packaging", Electronic Components and Technology Conference, Proceedings. 52nd.

[8] Wong, E. H., 2015, "The fundamentals of thermal-mass diffusion analogy", Microelectronics Reliability, Vol. 55, pp. 588-595.

[9] Wong, E. H. and Park, B., 2016, "Moisture diffusion modeling - a critical review", Microelectronics Reliability, Vol. 65, pp. 318-326.

[10] Silling S. A., 2000, "Reformulation of Elasticity Theory for Discontinuities and Long-range Forces", Journal of the Mechanics and Physics of Solids, Vol. 48, pp. 175209.

[11] Madenci, E. and Oterkus, E., 2014, "Peridynamic Theory and Its Applications", Springer, New York.

[12] Oterkus, S., Madenci, E. and Agwai, A., 2014, "Peridynamic thermal diffusion", Journal of Computational Physics, Vol. 265, pp. 71-96.

[13] Jang, C., Park, S., Han, B., Yoon, S., 2008, “Advanced Thermal-Moisture Analogy Scheme for Anisothermal Moisture Diffusion Problem", Journal of Electronic Packaging, Vol. 130, pp.11004. 